\title{
Discrete gravity and its continuum limit
}

\author{
Yi $\operatorname{Ling}^{1,2 *}$ \\ 1 CCAST (World Laboratory), P.O. Box 8730, Beijing 100080, China and \\ 2 Institute of Theoretical Physics, Chinese Academy of Sciences, \\ P.O.Box 2735, Beijing 100080, China
}

\begin{abstract}
Recently Gambini and Pullin proposed a new consistent discrete approach to quantum gravity and applied it to cosmological models. One remarkable result of this approach is that the cosmological singularity can be avoided in a general fashion. However, whether the continuum limit of such discretized theories exists is model dependent. In the case of massless scalar field coupled to gravity with $\Lambda=0$, the continuum limit can only be achieved by fine tuning the recurrence constant. We regard this failure as the implication that cosmological constant should vary with time. For this reason we replace the massless scalar field by Chaplygin gas which may contribute an effective cosmological constant term with the evolution of the universe. It turns out that the continuum limit can be reached in this case indeed.
\end{abstract}

PACS numbers: 04.60.Nc, 04.20.Dw, 04.60.Kz

*Electronic address: yling@itp.ac.cn 


\section{INTRODUCTION}

The cosmological singularity is a longstanding and fundamental problem in quantum cosmology. Nowadays both string theory and loop quantum gravity have been taking this issue into account seriously, see 1, 2] and [3] for recent progress along both approaches respectively. Motivated from string theory, the cosmological solution from big crunch to big bang has been proposed in [1], which can also be viewed as the orbifold compactification of solutions in higher dimensional field theory. This solution has very peculiar features. For instance it can be viewed as the Milne universe but identified by the boost such that it contains closed timelike loops and more badly, not Hausdorff. On the other hand this solution plays a special role in string/M theory since such spacetime is flat everywhere except at the singularity and the scalar field keeps staying in the weak coupling region while approaching to the singularity. Consequently the behavior of strings nearby such singularity in context of string/M theory has been studied. Although some positive results have been

obtained in 2], implying strings may pass through such singularity, the instability of this solution was also pointed out in [4]. Therefore further delicate consideration is needed to the understanding of cosmological singularity in string theory.

Recently another novel program called as discrete gravity has shed new light on this problem [5] as well. The idea is to discretize Einstein equations. But in contrast to usual lattice quantum gravity with fixed multipliers which breaks diffeomorphism invariance manifestly, in discrete gravity the lagrangian multipliers have to vary with "time" level $n$ such that discretized equations can be preserved consistent. Furthermore, unlike ordinary mechanism of canonical quantum gravity, discrete gravity contains an attractive feature of constraint free such that many conceptual problems like the problem of time might be solved in a more promising way[6]. In context of cosmology this program has also appealing advantages of evolving through the Big-Bang singularity in a general fashion and naturally introducing a notion of time at quantum mechanical level.

In this paper we apply the discrete techniques to study the cosmological model which describes the universe may evolve from a big crunch to a big bang. In this context we will see our results support the argument that the cosmological singularity can be avoided in a general fashion. But with a distinct starting point from [5], we prefer to considering the fate of singularities in universes with $\Lambda=0$ because in the case of $\Lambda \neq 0$, the classical 
singularities in vacuum are often coordinate singularities in the absence of matter, although this claim does not mean that the singularity at quantum mechanical level must not genuine. The discrete theory of FRW cosmology with $\Lambda=0$ has been considered in [5]. However, as its authors pointed out, the discrete theory describing the universe with $\Lambda=0$ often suffers from the absence of the continuum limit, which has negative implications to the quantization of the theory. We will also meet this trouble in next section when the model with massless scalar field is considered. One purpose of our paper is to see whether we may improve the continuum behavior of the discrete theory with zero cosmological constant. As a way coming out, we consider the universe dominated by Chaplygin gas rather than the ordinary massless scalar field in section four. Such an exotic fluid behaves as matter dust at early time, but contribute a nonzero effective cosmological constant at later time.

We organize the paper as follows. In next section we briefly review the framework of discrete mechanics focusing on constrained system. Then we turn to consider the model with a massless scalar field with $\Lambda=0$ in section three. After discussing the relations between solutions in discretized theory and those in continuum field theory, we argue that the singularity may be avoided in discrete cosmology. In section four we study Chaplygin gas dominated universe to obtain a better continuum limit of such universes with vanishing cosmological constant at early time.

\section{DISCRETE THEORY OF CONSTRAINED SYSTEM}

In this section we briefly review the classical framework of discrete theory of constrained system which we will use in next sections. For more details and in particular the quantization of discrete system we refer to [5, 6]. We start with the action of a continuum mechanical system

$$
S=\int d t L_{c}(q, \dot{q})
$$

The discretized formalism is implemented by splitting time into equal intervals $t_{n}=n \epsilon$ where $\epsilon$ is an infinitesimal constant. As a result, all the derivatives with respect to time are replaced by discrete first order finite difference,

$$
q=q_{n}, \quad \dot{q}=\frac{q_{n+1}-q_{n}}{\epsilon} .
$$


The time integral in the action then is replaced by a sum $S=\sum_{n=1}^{N} L\left(q_{n}, q_{n+1}\right)$, where $L\left(q_{n}, q_{n+1}\right)$ is understood as the discretized Lagrangian at instant $n$ with relations $L\left(q_{n}, q_{n+1}\right)=\epsilon L_{c}(q, \dot{q})$.

For a constrained system, we can straightforwardly obtain $L\left(q_{n}, q_{n+1}\right)$ from $L_{c}(q, \dot{q})$ with the form,

$$
\begin{aligned}
L\left(q_{n}, q_{n+1}\right) & \equiv L(n, n+1) \\
& =p_{n}\left(q_{n+1}-q_{n}\right)-\epsilon H\left(q_{n}, p_{n}\right)-\lambda_{n} C\left(q_{n}, p_{n}\right),
\end{aligned}
$$

where $H$ is the discrete version of Hamiltonian and $C$ denotes the constraint. Note in the last term the parameter $\epsilon$ has been absorbed into $\lambda_{n}$. In this form we have also defined the canonically conjugate momenta as

$$
p_{n+1}=\frac{\partial L\left(q_{n}, q_{n+1}\right)}{\partial q_{n+1}} .
$$

In terms of canonical conjugate pairs the equations of motion can be written as,

$$
\begin{aligned}
P_{n+1}^{q}-P_{n}^{q} & =-\epsilon \frac{\partial H\left(q_{n}, P_{n+1}^{q}\right)}{\partial q_{n}}-\lambda_{n} \frac{\partial C\left(q_{n}, P_{n+1}^{q}\right)}{\partial q_{n}} \\
q_{n+1}-q_{n} & =\epsilon \frac{\partial H\left(q_{n}, P_{n+1}^{q}\right)}{\partial P_{n+1}^{q}}+\lambda_{n} \frac{\partial C\left(q_{n}, P_{n+1}^{q}\right)}{\partial P_{n+1}^{q}} \\
C\left(q_{n}, P_{n+1}^{q}\right) & =0 .
\end{aligned}
$$

One significant difference between these equations and those continuum ones is that in discrete theory the lagrangian multipliers have to be fixed at each instant in order for the discrete version of the "constraints" to be preserved.

Therefore given a continuum theory with constraints we may obtain its discrete Lagrangian and equations of motion. Going further along this approach, we have two important issues to consider. One is to investigate whether the discrete theory may give rise to any different prediction comparing with the continuum theory. The other one is to check whether the continuum theory can be recovered by taking the continuum limit of the discrete theory. In next sections we will apply this framework to cosmology and see these cosmological models may help us to clarify these questions. 


\section{BIG CRUNCH/BANG UNIVERSE}

Consider a general 4d theory of a scalar field coupled to gravity. The Lagrangian has the form

$$
S=\int_{M}\left(R \sqrt{-g}-\frac{1}{2} g^{\mu \nu} \partial_{\mu} \phi \partial_{\nu} \phi-V(\phi)\right),
$$

where we use the $(-,+,+,+)$ signature for spacetime. In context of cosmology we adopt minisuperspace description. The metric reduces in conformal coordinate system to

$$
d s^{2}=a^{2}(t)\left[-N^{2}(t) d t^{2}+d x_{i}^{2}\right]
$$

where $a$ is the scale factor and $N$ lapse function. We strict our attention in four dimensional spacetime with vanishing $V(\phi)$ in this section such that the lagrangian has the form

$$
\mathcal{L}=\frac{-1}{N}\left(4 \dot{a}^{2}-\frac{1}{3} a^{2} \dot{\phi}^{2}\right)
$$

The cosmological solution from big crunch to big bang has the form [1],

$$
a(t)=a(0)|t|^{\frac{1}{2}} \quad \phi(t)=\phi(0)+\sqrt{3} \ln |t|,
$$

where $t$ is from $-\infty$ to $+\infty$. Obviously this solution has a singularity at $a=0$ and $\phi=-\infty$ as $t$ goes to zero. Such a singularity implies that the lagrangian breaks down near the neighborhood of that point. As in the quantum or semiclassical theory of hydrogen whose apparently continued spectrum is actually discrete at microscopic level, we propose the similar consideration should be applicable to the semiclassical theory of spacetime geometry. [12] As a result we conjecture a discretized version of lagrangian (8) probably is a good starting point to better understand the microscopic origin of the universe. The classical solution (9) may be only valid macroscopically, but should be achieved by taking continuum limit of solutions in the discretized theory.

First consider the canonical formalism of the theory. We define conjugate momenta to scalar factor $a(t)$ and $\phi(t)$,

$$
\pi_{a}=\frac{\partial \mathcal{L}}{\partial \dot{a}}=\frac{-8 \dot{a}}{N}, \quad \pi_{\phi}=\frac{\partial \mathcal{L}}{\partial \dot{\phi}}=\frac{2 a^{2} \dot{\phi}}{3 N},
$$

while the conjugate momentum of lapse function $N, \pi_{N}$, is zero. The hamiltonian can be constructed as

$$
\mathcal{H}=\pi_{a} \dot{a}+\pi_{\phi} \dot{\phi}-\mathcal{L}=\frac{-N}{4}\left(\frac{\pi_{a}^{2}}{4}-\frac{3 \pi_{\phi}^{2}}{a^{2}}\right) \equiv N H
$$


The discrete version of an action is obtained from (3)

$$
\begin{aligned}
L(n, n+1) & =\pi_{\phi n}\left(\phi_{n+1}-\phi_{n}\right)+\pi_{a n}\left(a_{n+1}-a_{n}\right) \\
& +\frac{N_{n}}{4}\left(\frac{\pi_{a n}^{2}}{4}-\frac{3 \pi_{\phi n}^{2}}{a_{n}^{2}}\right),
\end{aligned}
$$

where $\epsilon$ has been absorbed into the multiplier $N_{n}$.

From (4) one find the momenta at instants $n$ and $n+1$ respectively are

$$
\begin{array}{ll}
P_{n+1}^{\phi}=\pi_{\phi n} & P_{n}^{\phi}=\pi_{\phi n} \\
P_{n+1}^{\pi_{\phi}}=0 & P_{n}^{\pi_{\phi}}=-\left(\phi_{n+1}-\phi_{n}\right)+\frac{3 N_{n} \pi_{\phi n}}{2 a_{n}^{2}} \\
P_{n+1}^{a}=\pi_{a n} & P_{n}^{a}=\pi_{a n}-\frac{3 N_{n} \pi_{\phi n}^{2}}{2 a_{n}^{3}} \\
P_{n+1}^{\pi_{a}}=0 & P_{n}^{\pi_{a}}=-\left(a_{n+1}-a_{n}\right)-\frac{N_{n} \pi_{a n}}{8} \\
P_{n+1}^{N}=0 & P_{n}^{N}=\frac{1}{4}\left(\frac{\pi_{a n}^{2}}{4}-\frac{3 \pi_{\phi n}^{2}}{a_{n}^{2}}\right) .
\end{array}
$$

Taking $\left(a_{n}, \phi_{n}, P_{n+1}^{a}, P_{n+1}^{\phi}\right)$ as the fundamental variables yields the following compact equations

$$
\begin{aligned}
P_{n+1}^{\phi}-P_{n}^{\phi} & =0 \\
P_{n+1}^{a}-P_{n}^{a} & =\frac{3 N_{n} \pi_{\phi n}^{2}}{2 a_{n}^{3}} \\
\phi_{n+1}-\phi_{n} & =\frac{3 N_{n} \pi_{\phi n}}{2 a_{n}^{2}} \\
a_{n+1}-a_{n} & =\frac{-N_{n} \pi_{a n}}{8} \\
\frac{\left(P_{n+1}^{a}\right)^{2}}{4} & =\frac{3\left(P_{n+1}^{\phi}\right)^{2}}{a_{n}^{2}} .
\end{aligned}
$$

We see $P_{n}^{\phi}$ is a conserved quantity independent of level $n$. For convenience we may set it as a positive constant $C$. Plugging it into the last equation, we have

$$
P_{n+1}^{a}= \pm \frac{2 \sqrt{3} C}{a_{n}} .
$$

Then from the other equations in (14) it's straightforward to obtain the recurrence relation of the scale factor as

$$
\frac{a_{n+1}}{a_{n}}=\frac{a_{n}}{a_{n-1}} \equiv q,
$$


where $q$ can be any positive constant. Given a fixed $q$ and initial conditions, we have two kinds of solutions corresponding to the different signs of $P_{n+1}^{a}$ in (15).

$$
\begin{array}{rlrl}
a_{n} & \sim a_{0} q^{n}, & \phi_{n} \sim \phi_{0}+2 \sqrt{3} n(q-1) \\
N_{n} \sim \frac{4 a_{0}^{2} q^{2 n}(q-1)}{\sqrt{3} C}, & P_{n+1}^{a} \sim-2 \sqrt{3} \frac{C}{a_{n}}
\end{array}
$$

or

$$
\begin{array}{lll}
a_{n} \sim a_{0}^{\prime} q^{\prime n}, & \phi_{n} \sim \phi_{0}^{\prime}+2 \sqrt{3} n\left(1-q^{\prime}\right) \\
N_{n} \sim \frac{4 a_{0}^{2} q^{\prime 2 n}\left(1-q^{\prime}\right)}{\sqrt{3} C^{\prime}}, & P_{n+1}^{a} \sim 2 \sqrt{3} \frac{C^{\prime}}{a_{n}} .
\end{array}
$$

One remarkable result from these solutions is that the lapse $N_{n}$ is determined at each instant by the equation, contrasting to the continuum theory where $N$ is a free Lagrangian multiplier and may be set to one uniformly. Unfortunately we notice from (16) that unless the factor $q$ is very close to one the continuum limit can not be achieved as the "time" level $n$ approaches to infinity. In particular the continuum solution can only be achieved with a certain accuracy as we choose the desired $q$. Nevertheless, this discrete solution may give rise to the classical solution given in (9). To see this we need set the lapse function to unity as $n$ approaches to infinity, otherwise the comparison is not meaningful. Consider the solutions (17) and note that

$$
\epsilon N \sim N_{n} \sim \frac{4 a_{n}\left(a_{n+1}-a_{n}\right)}{\sqrt{3} C}
$$

we take the continuum limit and set $N=1$, leading to

$$
a \dot{a} \sim 1
$$

whose solution is $a \sim t^{1 / 2}$. Here we have also defined that $t=n \epsilon$ and $\dot{a}=\frac{a_{n+1}-a_{n}}{\epsilon}$. If $q \sim e^{\delta}$ where $\delta$ is a small number, then we have $\delta \sim \frac{\ln (t)}{2 n}$, yielding the classical solution $\phi \sim \phi_{0}+\sqrt{3} \ln (t)$. Of course in this sense $q$ is not a strict constant any more but approaches to one as $n$ goes to infinity.

The cosmological solution from big crunch to big bang can be obtained by combining the solution (18) with $q^{\prime}<1$ when $n \leq 0$ and (17) with $q>1$ when $n \geq 0$. To be single valued at $n=0$, we have adjoint conditions $a_{0}=a_{0}^{\prime}, C=C^{\prime}, \phi_{0}=\phi_{0}^{\prime}$ and $q+q^{\prime}=2$. But we note that the conjugate momentum of $a_{n}$ has a jump from $\pi_{0^{-}}^{a^{\prime}}=2 \sqrt{3} \frac{C}{a_{0}}$ to $\pi_{0^{+}}^{a}=-2 \sqrt{3} \frac{C}{a_{0}}$. Physically it means the universe contracts as the time level $n$ increases from $-\infty$ until $n=0$ 
and then rebound at the minimum size of the universe, turning into an expanding phase as $n>0$. In this discretized version, we find the singularity as $\mathrm{t}$ approaches to zero is always avoided unless we set $a_{0}=0$ by hand. But in generic points $a_{0}$ should be determined by the evolution of scale factor from $n<0$ to $n=0$, which in classical limit corresponds to the evolution from $t=-\infty$ to $t=0$. As a matter of fact, all these intuitive considerations might be invalid as the gravitation effect becomes so strong that the quantum theory of gravity should be taken into account. Then the quantum states of the universe is the superpositions of such states describing expanding and contracting universes. Nevertheless based on this heuristic analysis we believe the evolution of universe becomes more delicate due to the quantum effect of gravity and rather than a genuine crunch occurs, it might rebound once a minimum size of the scale factor is saturated.

We realize once the quantization of discrete theory is concerned, we need a probability explanation of such special initial conditions, namely why the recurrence constant $q$ is close to one but not else. In classical theory we are short of such strategy to present a satisfying explanation. Based on above consideration, we see the closest argument appearing in cosmological model from big crunch to big bang is the requirement that $q+q^{\prime}=2$. If $a_{n}$ is always positive as it should be, then $q$ and $q^{\prime}$ could be any positive number in $(0,2)$, not directly leading to our expected value $e^{\delta}$ and $e^{-\delta}$ respectively. One might expect that the classical solution (16) is not stable unless the $q$ approaches to unity, in other words $q=1$ might be thought of as an attractor and such running of $q$ from any initial constant to unity might be implemented or required at quantum mechanical level. But following perturbative analysis shows it's not the case. Consider the perturbation

$$
\begin{aligned}
a_{n} & =a_{n}^{(0)}+\delta a_{n}, \quad \phi_{n}=\phi_{n}^{(0)}+\delta \phi_{n}, \\
P_{n}^{a} & =P_{n}^{a(0)}+\delta P_{n}^{a}, \quad P_{n}^{\phi}=P_{n}^{\phi(0)}+\delta P_{n}^{\phi} \\
N_{n} & =N_{n}^{(0)}+\delta N_{n} .
\end{aligned}
$$

Substituting into equations of motion (14) and keeping the first order correction, we have

$$
\delta a_{n+1}+\frac{2 q-8}{3} \delta a_{n}+q^{2} \delta a_{n-1}=0 .
$$

On the other hand, perturbations directly based on (16) leads to

$$
\delta a_{n+1}-2 q \delta a_{n}+q^{2} \delta a_{n-1}=0 .
$$


The consistency requires that $q \sim 1$, which looks satisfying. But another kind of reasonable perturbation is $a_{n}=a_{0}(q+\delta q)^{n}$ such that $\delta a_{n+1}=\frac{n+1}{n} q \delta a_{n}$. It immediately leads to the conclusion that solutions (17) and (18) are classically stable except for $q=1$, at that point $\delta a_{n}$ could be any value, which is opposite to what we desire.

We hope this dilemma may be solved by further progress in the quantum theory of gravity. But before that we want to present another model where a good continuum limit can be achieved nearby the singularity in next section.

\section{CHAPLYGIN GAS}

Chaplygin gas as an exotic fluid has been greatly investigated in FRW cosmological model [7, 8] ( also see [8] for more references). Since it behaves like a cosmological constant at later time of the universe, namely contributing a positive energy density and negative pressure, it is supposed to be responsible for the acceleration of the universe, as indicated by recent observation[9]. But at early time of the universe it behaves just like a dust matter such that the effective cosmological constant is zero. One interesting feature of Chaplygin gas is that its lagrangian is quite similar to the effective lagrangian of tachyon field in string theory, which has been proposed by A. Sen[10], even though the exact expression is not clear yet. The lagrangian of both Chaplygin gas and tachyon fields can be given as

$$
\mathcal{L}=\frac{-1}{2 N}\left(\dot{a}^{2}+\frac{8 \pi G a^{4} N^{2} V}{3} \sqrt{1-\frac{\dot{T}^{2}}{N^{2} a^{2}}}\right)
$$

For Chaplygin gas, the potential $V(T)$ is a constant, while for tachyon fields $V(T)$ has a maximum at $T=0$ and exponentially decreases for large $T[13]$.

In this section we consider the discrete theory of Chaplygin cosmology. To compare our result with those in previous section, we still adopt the conformal coordinate system

$$
d s^{2}=a^{2}(t)\left[-N^{2}(t) d t^{2}+d x_{i}^{2}\right]
$$

As a matter of fact, we are free to redefine the lapse function,

$$
N^{\prime}(t):=N(t) a(t)
$$

and then turn to

$$
d s^{2}=-N^{\prime 2}(t) d t^{2}+a^{2}(t) d x_{i}^{2}
$$


The equations of motion and constraints have the following familiar form

$$
\begin{aligned}
& \frac{1}{a N^{\prime}} \frac{d}{d t}\left(\frac{\dot{a}}{N^{\prime}}\right)=\frac{8 \pi G}{3} \frac{V(T)}{\sqrt{1-\frac{\dot{T}^{2}}{N^{\prime 2}}}}\left(1-\frac{3 \dot{T}^{2}}{2 N^{\prime 2}}\right) \\
& \frac{\dot{a}^{2}}{a^{2} N^{\prime 2}}=\frac{8 \pi G}{3} \frac{V(T)}{\sqrt{1-\frac{\dot{T}^{2}}{N^{\prime 2}}}} \\
& \frac{N^{\prime}}{N^{\prime 2}-\dot{T}^{2}} \frac{d}{d t}\left(\frac{\dot{T}}{N^{\prime}}\right)+3 \frac{\dot{a} \dot{T}}{a N^{\prime 2}}+\frac{1}{V} \frac{d V}{d T}=0 .
\end{aligned}
$$

As is familiar, we call the first two equations as Raychaudhuri and Friedman equations respectively in standard cosmology and the third one is the equation of motion for tachyon fields. Furthermore, as these three equations are not independent we are free to set the lapse function $N^{\prime}=1$.

Next we consider the canonical formalism of the theory. Define the conjugate momenta of scale factor $a$ and scalar field $T$

$$
\begin{aligned}
& \pi_{a}=\frac{\partial \mathcal{L}}{\partial \dot{a}}=\frac{-\dot{a}}{N} \\
& \pi_{T}=\frac{\partial \mathcal{L}}{\partial \dot{T}}=\frac{4 \pi G}{3} \frac{a^{3} V \dot{T}}{\sqrt{N^{2} a^{2}-\dot{T}^{2}}}
\end{aligned}
$$

then the Hamiltonian is given as

$$
\begin{aligned}
\mathcal{H} & =\pi_{a} \dot{a}+\pi_{T} \dot{T}-\mathcal{L} \\
& =\frac{-1}{2 N}\left(\dot{a}^{2}-\frac{8 \pi G a^{4} N^{2}}{3} \frac{V}{\sqrt{1-\frac{\dot{T}^{2}}{N^{2} a^{2}}}}\right) .
\end{aligned}
$$

The discretized version of the lagrangian is

$$
\begin{aligned}
L(n, n+1) & =\pi_{a n}\left(a_{n+1}-a_{n}\right)+\pi_{T n}\left(T_{n+1}-T_{n}\right) \\
& +\frac{N_{n}}{2}\left[\pi_{a n}^{2}-2 a_{n} \sqrt{\pi_{T n}^{2}+\left(\frac{4 \pi G a_{n}^{3} V}{3}\right)^{2}}\right] .
\end{aligned}
$$

The canonically conjugate momenta of these variables at instant $n+1$ and $n$ are respectively 
given by

$$
\begin{aligned}
P_{n+1}^{a}=\pi_{a n} & P_{n}^{a}=\pi_{a n}+N_{n}\left[\lambda_{n}+\left(\frac{4 \pi G V}{3}\right)^{2} \frac{3 a_{n}^{6}}{\lambda_{n}}\right] \\
P_{n+1}^{\pi_{a}}=0 & P_{n}^{\pi_{a}}=-\left[\left(a_{n+1}-a_{n}\right)+N_{n} \pi_{a n}\right] \\
P_{n+1}^{\pi_{T}}=0 & P_{n}^{\pi_{T}}=-\left[\left(T_{n+1}-T_{n}\right)-\frac{N_{n} a_{n} \pi_{T n}}{\lambda_{n}}\right] \\
P_{n+1}^{T}=\pi_{T n} & P_{n}^{T}=\pi_{T n}+\left(\frac{4 \pi G a_{n}^{3}}{3}\right)^{2} \frac{N_{n} a_{n} V}{\lambda_{n}} \frac{d V}{d T} \\
P_{n+1}^{N}=0 & P_{n}^{N}=\frac{1}{2}\left(\pi_{a n}^{2}-2 a_{n} \lambda_{n}\right),
\end{aligned}
$$

where we have defined

$$
\lambda_{n} \equiv \sqrt{\pi_{T n}^{2}+\left(\frac{4 \pi G a_{n}^{3} V}{3}\right)^{2}} .
$$

In discrete theory the time evolution is described by a canonical transformation from the variables from $\left(q_{n}, p_{n}\right)$ to $\left(q_{n}, p_{n+1}\right)$. Taking $\left(a_{n}, T_{n}, P_{n}^{a}, P_{n}^{T}\right)$ as the set of basic variables, we rewrite these equations of motion as

$$
\begin{aligned}
& a_{n+1}-a_{n}=-N_{n} \pi_{a n} \\
& P_{n+1}^{a}-P_{n}^{a}=-N_{n}\left[\lambda_{n}+3\left(\frac{4 \pi G V}{3}\right)^{2} \frac{a_{n}^{6}}{\lambda_{n}}\right] \\
& T_{n+1}-T_{n}=\frac{N_{n} a_{n} \pi_{T n}}{\lambda_{n}} \\
& P_{n+1}^{T}-P_{n}^{T}=-\left(\frac{4 \pi G a_{n}^{3}}{3}\right)^{2} \frac{N_{n} a_{n} V}{\lambda_{n}} \frac{d V}{d T} \\
& \left(P_{n+1}^{a}\right)^{2}-2 a_{n} \lambda_{n}=0
\end{aligned}
$$

Above consideration is applicable to both Chaplygin gas and tachyon field. From now on we only focus on the case of Chaplygin gas which has a constant $V$. The immediate consequence from above equation is that $P_{n+1}^{T}$ is conserved, which is nothing but $\pi_{T n}$. First we consider the expanding universe with very small scale factor comparing with $\pi_{T n}$,

$$
\pi_{T n} \gg\left(\frac{4 \pi G a_{n}^{3} V}{3}\right)
$$


then $\lambda_{n} \simeq \pi_{T n}=P_{n+1}^{T}$ and the equations of motion reduce to

$$
\begin{aligned}
& a_{n+1}-a_{n}=-N_{n} \pi_{a n} \\
& P_{n+1}^{a}-P_{n}^{a}=-N_{n} \pi_{T n} \\
& T_{n+1}-T_{n}=N_{n} a_{n} \\
& P_{n+1}^{T}-P_{n}^{T}=0 \\
& \left(P_{n+1}^{a}\right)^{2}-2 a_{n} P_{n+1}^{T}=0 .
\end{aligned}
$$

After solving for $N_{n}$ and $P_{n}^{a}$ from the second and fifth equation, we may obtain the recurrence relations of $a_{n}$ from the first equation as

$$
a_{n+1}^{2}-6 a_{n+1} a_{n}+9 a_{n}^{2}-4 a_{n} a_{n-1}=0 .
$$

As we mentioned the evolution scheme is given by the canonical transformation from $n$ to $n+1$. Given the initial conditions, the value of scale factor can be determined at any instant by the recurrence relation (37). In particular, whether the universe expands or contracts depends only on these initial data. For instance if we take $a_{1}>a_{0}$, then we will see $a_{n+1}>a_{n}$ for any $n>1$, implying an expanding universe. On the other side, if we choose $a_{-1}>a_{0}$, then we find $a_{n+1}<a_{n}$ for any $n<-1$, hence implying an contracting universe. Similar to the discussion in previous section, the universe from big crunch to big bang may be obtained by connecting these two solutions at $n=0$. We may also plot graphs to illustrate the discrete evolution of $a$ by choosing appropriate parameters such that the scale factor goes to zero as $n$ approaches to 0 from both $n<0$ and $n>0$, implying a singularity at $t=0$ in the continuum limit. But in discrete theory more detailed analysis indicates that scale factor takes a small but non-zero value at $n=0$ in general. Thus its solutions may also be used to describe a universe from big crunch to big bang but avoiding the singularity.

We remark that it is a conjecture that the universe from big crunch to big bang may be obtained by connecting two solutions at singularity, but not a simple solution to classical equations 1]. In our discrete theory it says that the set of $\left(a_{-1}, a_{0}, a_{1}\right)$ does not satisfy the classical equations (16) or (37). The dynamical explanation of how the universe rebounds at the minimum size $a_{0}$ is absent in this classical framework, but it is not unreasonable as we expect the quantum theory of discrete gravity would play an essential role to solve this issue. 
Now we turn to consider the continuum limit as $n$ goes to infinity. This can be done by recovering the first and second order derivatives with respect to time through

$$
\begin{aligned}
& \dot{a}=\frac{a_{n+1}-a_{n}}{\epsilon} \\
& \ddot{a}=\frac{a_{n+1}-2 a_{n}+a_{n-1}}{\epsilon^{2}} .
\end{aligned}
$$

Then equation (37) becomes

$$
\dot{a}^{2}-4 a \ddot{a}=0 .
$$

The general solution to this equation is

$$
a=c_{1}\left(t+c_{2}\right)^{\frac{4}{3}}
$$

where $c_{1}$ and $c_{2}$ are two constants, viewed as a remnant of the reparameterization invariance of the continuum theory. Now going back to the other equations in (36) and taking the continuum limit we straightforwardly obtain the asymptotical behavior of other parameters

$$
\pi_{a n} \sim t^{2 / 3} \quad N_{n} \sim t^{-1 / 3} \quad T \sim t^{2}
$$

and thus the metric has the form

$$
d s^{2} \sim t^{8 / 3}\left(-t^{-2 / 3} d t^{2}+d x_{i}^{2}\right)
$$

To see Chaplygin gas may be though of as a dust matter during this epoch we turn to the following conventional coordinate system with lapse function $N^{\prime}=1$ :

$$
d s^{2} \sim-d T^{2}+T^{4 / 3} d x_{i}^{2}
$$

Therefore the "effective" scale factor behaves as $a^{\prime} \sim T^{2 / 3}$, indicating an expanding but decelerating universe as $T>0$. One interesting point here is that during the origin of the universe the Chaplygin gas field may be understood as the proper time of the universe. Such kind of behavior is something like the time reversal of tachyon field, as pointed out in [11], where tachyon may play a role of time variable as rolling down to infinity at later time of the universe.

To see our discrete scheme actually leads to the same physics as the continuum field theory of Chaplygin gas, we may also consider the continuum limit for large scale factor. In this case we have,

$$
\pi_{T n} \ll\left(\frac{4 \pi G a^{3} V}{3}\right) .
$$


Since $V$ is a constant, then

$$
\lambda_{n} \simeq \frac{4 \pi G a^{3} V}{3}
$$

and the equations reduce to

$$
\begin{aligned}
& a_{n+1}-a_{n}=-N_{n} \pi_{a n} \\
& P_{n+1}^{a}-P_{n}^{a}=-4 N_{n} \lambda_{n} \\
& T_{n+1}-T_{n}=\frac{N_{n} a_{n} \pi_{T n}}{\lambda_{n}} \\
& P_{n+1}^{T}-P_{n}^{T}=0 \\
& \left(P_{n+1}^{a}\right)^{2}-2 a_{n} P_{n+1}^{T}=0 .
\end{aligned}
$$

Similarly we obtain the recurrence relation of $a_{n}$

$$
2 a_{n} a_{n+1}-3 a_{n}^{2}+a_{n-1}^{2}=0 .
$$

Taking the continuum limit yields the differential equation

$$
2 a \ddot{a}+\dot{a}^{2}=0 .
$$

One solution to this equation is $a \sim t^{2 / 3}$. Substituting it back to (46) we obtain the asymptotical behavior of other variables as follows

$$
\pi_{a n} \sim t^{4 / 3} \quad N_{n} \sim t^{-5 / 3} \quad T \sim t^{-2}
$$

As a result, the metric becomes

$$
d s^{2} \sim-\frac{1}{T^{2}} d T^{2}+T^{-2 / 3} d x_{i}^{2}
$$

We note that the Chaplygin gas does not play a role of proper time any more. In effect in the coordinate system with $N^{\prime}=1$ the metric goes to

$$
d s^{2} \sim-d \tau^{2}+\exp \frac{4}{3} \tau d x_{i}^{2}
$$

It indicates the universe is accelerating, thus with a positive cosmological constant, which is consistent with the result of continuum theory of Chaplygin gas. 


\section{CONCLUSIONS}

We have applied discrete gravity theory to investigate the universes from big crunch to big bang, mainly concerning the following two issues. One is on the fate of cosmological singularity. We proposed an attractive point of view on this issue based on the consistent discretization approach to general relativity. On the other hand we investigated the continuum limit of discrete theories describing the universe with vanishing cosmological constant. It's clear one may take the attempts presented here as the first step to understand what role the discrete gravity would play in quantum gravity and cosmology. In particular in this paper we leave all the considerations on the quantization of discrete gravity for further investigation. We expect the approach to discrete general relativity will show more insight into the problem of the cosmological singularity and quantum gravity.

\section{Acknowledgement}

I would like to thank Jorge Pullin and Lee Smolin for correspondence and helpful discussions. This work is partly supported by NSFC (No.10205002), SRF for ROCS, SEM and K.C.Wong Education Foundation, Hong Kong.

[1] J. Khoury, B. A. Ovrut, N. Seiberg, P. J. Steinhardt and N. Turok, Phys. Rev. D 65, 086007 (2002) arXiv:hep-th/0108187.

N. Seiberg, arXiv:hep-th/0201039.

H. Liu, G. Moore and N. Seiberg, arXiv:gr-qc/0301001.

[2] H. Liu, G. Moore and N. Seiberg, JHEP 0206, 045 (2002) arXiv:hep-th/0204168.

H. Liu, G. Moore and N. Seiberg, JHEP 0210, 031 (2002) arXiv:hep-th/0206182.

[3] M. Bojowald, Phys. Rev. Lett. 87, 121301 (2001) arXiv:gr-qc/0104072.

[4] G. T. Horowitz and J. Polchinski, Phys. Rev. D 66, 103512 (2002) arXiv:hep-th/0206228.

[5] R. Gambini and J. Pullin, Phys. Rev. Lett. 90, 021301 (2003) arXiv:gr-qc/0206055; C. Di Bartolo, R. Gambini and J. Pullin, Class. Quant. Grav. 19, 5275 (2002) arXiv:gr-qc/0205123;

R. Gambini and J. Pullin, Class. Quant. Grav. 20, 3341 (2003) arXiv:gr-qc/0212033.

[6] R. Gambini, R. A. Porto and J. Pullin, arXiv:gr-qc/0302064. 
[7] A. Y. Kamenshchik, U. Moschella and V. Pasquier, Phys. Lett. B 511, 265 (2001) arXiv:gr-qc/0103004.

H. B. Benaoum, arXiv:hep-th/0205140

[8] G. W. Gibbons, Class. Quant. Grav. 20, S321 (2003) arXiv:hep-th/0301117.

[9] S. Perlmutter et al. [Supernova Cosmology Project Collaboration], Bull. Am. Astron. Soc. 29, 1351 (1997) arXiv:astro-ph/9812473.

S. Perlmutter et al. [Supernova Cosmology Project Collaboration], Astrophys. J. 517, 565 (1999) arXiv:astro-ph/9812133.

[10] A. Sen, JHEP 0204, 048 (2002) arXiv:hep-th/0203211.

A. Sen, Mod. Phys. Lett. A 17, 1797 (2002) arXiv:hep-th/0204143.

[11] A. Sen, Int. J. Mod. Phys. A 18, 4869 (2003) arXiv:hep-th/0209122.

[12] This proposal has been strongly supported by loop quantum gravity, where the spectra of the area and volume operator are obtained at least at kinematical level and always taking discrete values.

[13] In particular, in [11] Sen conjectured that the tachyon field may not be physical matter, but viewed as the time variable in quantum cosmology as $\phi$ goes large. This interesting treatment has its advantages in quantum cosmology and more implications in nonperturbative quantum gravity may be further studied and discussed elsewhere. 